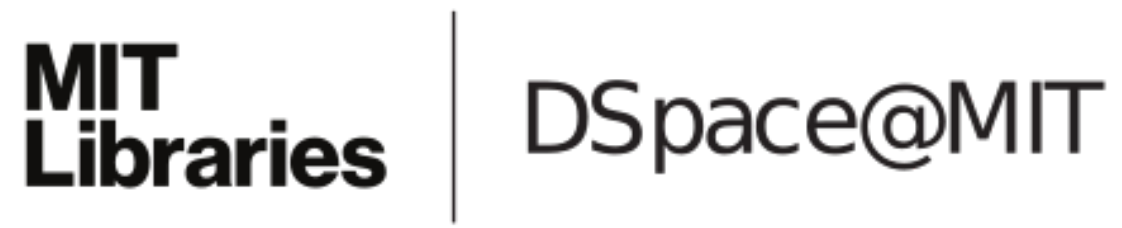

\author{
MIT Open Access Articles
}

\begin{abstract}
Understanding of hydrogen silsesquioxane electron resist for sub-5-nm-half-pitch lithography
\end{abstract}

The MIT Faculty has made this article openly available. Please share how this access benefits you. Your story matters.

Citation: Yang, Joel K. W. et al. "Understanding of Hydrogen Silsesquioxane Electron Resist for Sub-5-nm-half-pitch Lithography." Journal of Vacuum Science \& Technology B: Microelectronics and Nanometer Structures 27.6 (2009): 2622. (c) 2009 American Vacuum Society

As Published: http://dx.doi.org/10.1116/1.3253652

Publisher: American Vacuum Society (AVS)

Persistent URL: http://hdl.handle.net/1721.1/73050

Version: Final published version: final published article, as it appeared in a journal, conference proceedings, or other formally published context

Terms of Use: Article is made available in accordance with the publisher's policy and may be subject to US copyright law. Please refer to the publisher's site for terms of use. 


\title{
Understanding of hydrogen silsesquioxane electron resist for sub-5-nm-half-pitch lithography
}

\author{
Joel K. W. Yang, Bryan Cord, Huigao Duan, and Karl K. Berggren ${ }^{\text {a) }}$ \\ Department of Electrical Engineering and Computer Science, Massachusetts Institute of Technology, \\ Cambridge, Massachusetts 02139 \\ Joseph Klingfus \\ Raith USA, Inc., Ronkonkoma, New York 11779 \\ Sung-Wook Nam and Ki-Bum Kim \\ Department of Materials Science and Engineering, Seoul National University, Seoul 151742, South Korea \\ Michael J. Rooks \\ Department of Applied Physics, Yale University, New Haven, Connecticut 06520
}

(Received 14 July 2009; accepted 29 September 2009; published 2 December 2009)

\begin{abstract}
The authors, demonstrated that 4.5-nm-half-pitch structures could be achieved using electron-beam lithography, followed by salty development. They also hypothesized a development mechanism for hydrogen silsesquioxane, wherein screening of the resist surface charge is crucial in achieving a high initial development rate, which might be a more accurate assessment of developer performance than developer contrast. Finally, they showed that with a high-development-rate process, a short duration development of $15 \mathrm{~s}$ was sufficient to resolve high-resolution structures in 15 -nm-thick resist, while a longer development degraded the quality of the structures with no improvement in the resolution. (C) 2009 American Vacuum Society. [DOI: 10.1116/1.3253652]
\end{abstract}

\section{INTRODUCTION}

Electron-beam lithography (EBL) provides excellent patterning resolution: as demonstrated by electron-beam induced deposition (EBID) methods, patterns as small as 1.6-nm-half-pitch can be achieved. ${ }^{1}$ However, EBID methods are typically orders of magnitude slower due to the high exposure doses required and are less reproducible than resistbased processes. Therefore, EBID is less practical in patterning high-resolution structures over large areas. Furthermore, not all materials can be patterned by direct EBID processing.

On the other hand, the resist-based process using EBL exposure of hydrogen silsesquioxane (HSQ) resist is a promising approach for patterning high-resolution structures due to its higher speed (compared to EBID), high etch resistance of HSQ, and the convenience of pattern transfer from resist to various materials. In the past, we have demonstrated the patterning of 7-nm-half-pitch structures using this method, followed by a high-contrast salty-development step. ${ }^{2}$ This patterning capability has enabled several technologies such as the fabrication of high-resolution nanoimprint molds, ${ }^{3}$ narrow and densely packed suspended strained Si nanowires, ${ }^{4}$ and guiding structures for templating the self-assembly of block copolymers. ${ }^{5}$

Several other development techniques, such as hot development ${ }^{6-9}$ and $\mathrm{KOH}$ development, ${ }^{10}$ have been used to increase the contrast of HSQ with the premise that a higher contrast would result in a higher patterning resolution. Impressive high-resolution patterning of sub-20-nm pitch structures have been demonstrated using these methods. ${ }^{6,10}$ As the resolution of HSQ-based EBL is currently thought to be lim-

\footnotetext{
${ }^{a)}$ Electronic mail: berggren@mit.edu
}

ited in part by the resist performance, ${ }^{11}$ better understanding of HSQ should enable further improvements in the resolution.

Here we show that, while a high-contrast development process may be helpful, it is not an absolute indicator of the resolution performance of the process. For a typical resist system that exhibits a constant development rate with time, a high-contrast development process is expected to increase resolution while reducing the loss in resist thickness in the exposed regions. However, we show here that HSQ development is self-limiting in that its development rate becomes negligibly low with increasing development time. Hence, the relationship between contrast and resolution is less obvious in the case of HSQ development.

To explain these effects, we propose a hypothesis of HSQ development mechanism involving charge screening and suggest that a high initial development rate might be a more important parameter to consider than resist contrast. Finally, we demonstrate that a short duration development of $15 \mathrm{~s}$ was sufficient to fully resolve high-resolution structures.

\section{EXPERIMENTS AND RESULTS}

We first showed that 9-nm-pitch resolution could be achieved with a Raith 150-TWO EBL tool. ${ }^{12}$ To achieve this result, we spin coated a Si wafer with HSQ (FOx-12, Dow Corning) diluted with methyl-isobutyl ketone to achieve a thickness of $10 \mathrm{~nm}$. To avoid thermally induced contrast reduction, we did not bake the resist-coated wafer. Exposure was done at $10 \mathrm{kV}$ acceleration voltage, $20 \mu \mathrm{m}$ aperture, resulting in a beam current of $\sim 160 \mathrm{pA}$. Nested-"L" resolution test structures were exposed as single-pixel lines using a dose of $\sim 5 \mathrm{nC} / \mathrm{cm}(\sim 3000$ electrons $/ \mathrm{nm})$. The sample 

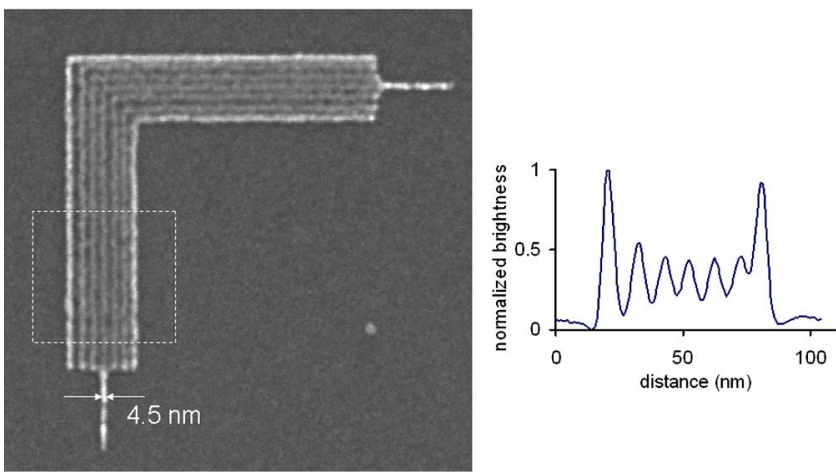

FIG. 1. (Color online) SEM image of 9-nm-pitch nested-L structures patterned in 10-nm-thick HSQ using the Raith $150^{\mathrm{TWO}}$ at $10 \mathrm{kV}$ acceleration voltage, followed by a 4 min development in aqueous $1 \mathrm{wt} \% \mathrm{NaOH}$, 4 wt $\% \mathrm{NaCl}$. SEM image was also taken using the same tool at $10 \mathrm{kV}$ acceleration voltage and a $7 \mathrm{~mm}$ working distance. The plot on the right was obtained by averaging the brightness value of the SEM image along the length of the line structures within the dashed box. We note that there might still be footing in between the structures that would need higher-resolution imaging methods to verify. Nonetheless, the clear modulation in the brightness signal of the SEM indicates that this new tool was capable of patterning at an impressive resolution of 4.5-nm-half-pitch. In this experiment, the vertical lines were marginally better resolved than the horizontal lines due to slight stigmation inaccuracies in the beam during exposure. The higher brightness of the outermost features is presumed to be due to greater facility in secondary-electron emission from the sides of these structures during imaging.

was then developed in aqueous $1 \mathrm{wt} \% \mathrm{NaOH}, 4$ wt $\% \mathrm{NaCl}$ in de-ionized (DI) water for $4 \mathrm{~min}$ at $24{ }^{\circ} \mathrm{C}$ developer temperature, followed by rinsing with DI water for at least $1 \mathrm{~min}$ and drying in a $\mathrm{N}_{2}$ stream. In all experiments presented here, development was done by sample immersion without agitation in a beaker containing the developer solution. Developments using ultrasonic agitation and manual agitation did not result in any observable difference, suggesting that the supply of fresh developer solution was the same in all cases. Care was taken to ensure that the rinse in DI water was long enough $(2 \mathrm{~min}$ ) to completely avoid any deposition of $\mathrm{NaCl}$ salt crystals on the surface of the substrate. Although we show below that $15 \mathrm{~s}$ development time was sufficient for resolving structures patterned in thin $(\sim 15 \mathrm{~nm})$ resists, we did not perform the short-development experiments during the limited time that we had access to the Raith 150-TWO.

Figure 1 shows a scanning electron micrograph (SEM) of a 4.5 -nm-half-pitch nested-L structure imaged at $10 \mathrm{kV}$ using the same tool used for the exposure. Patterning at $10 \mathrm{kV}$ instead of $30 \mathrm{kV}$ (the highest acceleration voltage on the Raith 150-TWO) speed up our exposures by a factor of $\sim 3$ without observable loss in resolution. Best efforts to achieve this level of patterning resolution on the Raith 150 EBL tool at the MIT campus resulted in 10-nm-pitch structures.

To study the effect of contrast on the resolution, we compared high-resolution test structures developed using three different developers: (1) $25 \mathrm{wt} \%$ tetramethyl ammonium hydroxide (TMAH developer), (2) $1 \mathrm{wt} \% \mathrm{NaOH}$ (nonsalty developer), and (3) $1 \mathrm{wt} \% \mathrm{NaOH}, 4 \mathrm{wt} \% \mathrm{NaCl}$ (salty developer). The contrast curves of these developers were measured and reported previously. ${ }^{2}$ The contrast values $\gamma$ for

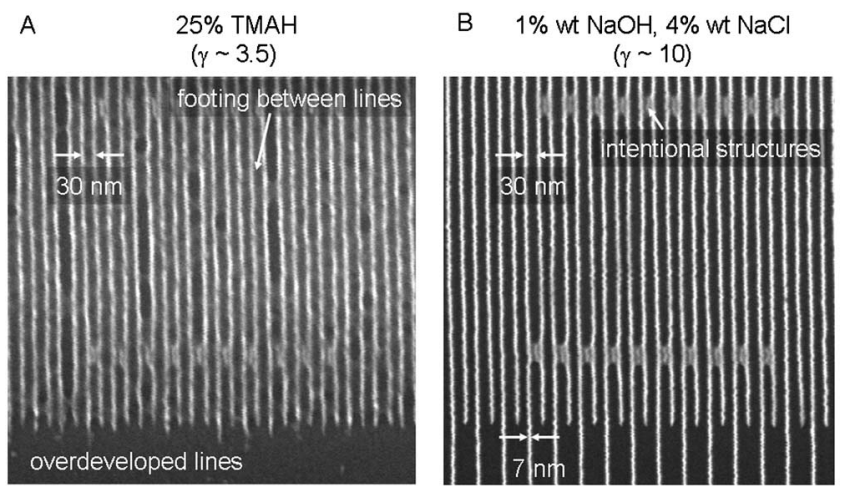

$25 \mathrm{~nm}$ thick HSQ

FIG. 2. SEM images of 30-nm-pitch structures that extend to a region of $60 \mathrm{~nm}$ pitch at the bottom of the image developed using (a) the 25\% TMAH developer and (b) the salty developer consisting of 1 wt $\% \mathrm{NaOH}, 4$ wt \% $\mathrm{NaCl}$. Imaging was done in a field emission SEM at $5 \mathrm{kV}$ acceleration voltage and $4 \mathrm{~mm}$ working distance. (a) With the low contrast $(\gamma \sim 3.5) 25 \%$ TMAH developer, footing/bridging formed in between the 30-nm-pitch lines where the resist was not completely developed. Furthermore, no process window existed where both 30-nm- and 60-nm-pitch lines resulted; the 60-nm-pitch lines were already overdeveloped before the resist could be cleared in between the 30-nm-pitch lines. The line dose for this pattern was $\sim 2 \mathrm{nC} / \mathrm{cm}$. (b) With the high-contrast $(\gamma \sim 10)$ salty developer, both $30-\mathrm{nm}$ and 60-nm-pitch lines resulted suggesting that a higher-contrast developer resulted in a larger process latitude. The line dose for this pattern was $\sim 7 \mathrm{nC} / \mathrm{cm}$.

these three developers were measured to be $\gamma \sim 3.5$ (TMAH developer), $\gamma \sim 3$ (nonsalty developer), and $\gamma \sim 10$ (salty developer) at a resist thickness of $115 \mathrm{~nm}$ and a development time of 4 min.

HSQ (XR-1541 2\% solids, Dow Corning) was spin coated onto $\mathrm{Si}$ substrates to a thickness of $25 \mathrm{~nm}$. The substrate was then cleaved into three pieces. Each sample was exposed in a Raith 150 EBL tool at $30 \mathrm{kV}$ acceleration voltage using a $30 \mu \mathrm{m}$ aperture, which resulted in a beam current of $\sim 400 \mathrm{pA}$. The exposure pattern consisted of single-pixelline gratings where half of the lines were extended to form a region of twice the pitch [see Fig. 2(b)]. The three samples were then developed in the three different developers mentioned above at $24{ }^{\circ} \mathrm{C}$, each for $4 \mathrm{~min}$, followed by rinsing with DI water for 1 min and blow drying with a $\mathrm{N}_{2}$ stream.

SEMs comparing the results of 30-nm- and 60-nm-pitch lines from the TMAH and salty developers are shown in Fig. 2. We observe a clear difference between the low-contrast $(\gamma \sim 3.5)$ TMAH developer and the high-contrast $(\gamma \sim 10)$ salty developer. With the salty developer, both $30-\mathrm{nm}$ and 60-nm-pitch regions were well defined. However, with the TMAH developer, the 30-nm-pitch region had severe footing/bridging between lines, while the $60-\mathrm{nm}$-pitch region was overdeveloped, indicating that the absence of a process window existed where both regions could be well defined.

Figure 3 shows SEMs comparing the results of $25-\mathrm{nm}$ and 50-nm-pitch lines from the nonsalty and salty developers. In stark contrast to the TMAH-salty developer comparison, here we observe only a slight difference between the low-contrast $(\gamma \sim 3)$ nonsalty and high-contrast $(\gamma \sim 10)$ salty developers. The only difference is that in the collapsed structures of the 

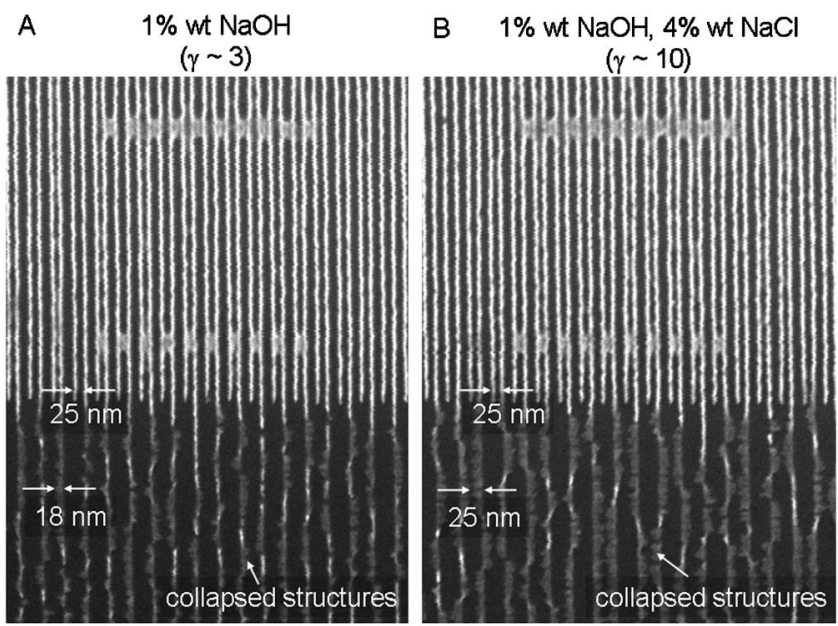

FIG. 3. SEM images of 25-nm-pitch structures that extended to a region of 50-nm-pitch structures at the bottom of the image patterned in 25-nm-thick HSQ using a line dose of $\sim 7 \mathrm{nC} / \mathrm{cm}$ and developed using (a) nonsalty 1 wt $\% \mathrm{NaOH}$ developer and (b) salty 1 wt $\% \mathrm{NaOH}, 4$ wt $\% \mathrm{NaCl}$ developer. Images were taken in a Zeiss SEM at $5 \mathrm{kV}$ acceleration voltage and $4 \mathrm{~mm}$ working distance. The structures in the 50-nm-pitch regions collapsed, which allowed us to measure the height of the remaining resist thickness by measuring the width of the collapsed structures. (a) Despite the significantly lower contrast $\gamma \sim 3$, the nonsalty developer was able to resolve the dense 25-nm-pitch lines. However, the lower contrast was evident in the greater loss in resist thickness compared to the salty development, which preserved the original resist thickness in the final structures.

50-nm-pitch regions, by measuring the width of the collapsed lines, we estimated that the salty development preserved the original resist thickness of $\sim 25 \mathrm{~nm}$, while the nonsalty development resulted in some loss in the resist thickness with only $\sim 18 \mathrm{~nm}$ of the resist remaining. This loss in the resist thickness at the nanoscale dimensions was also consistently observed in the microscale measurements of contrast curves: the contrast curves in Ref. 2 (Fig. 2) showed that the nonsalty developer resulted in greater loss in the resist thickness compared to the salty developer. Surprisingly though, even at $25 \mathrm{~nm}$ pitch, the low-contrast $(\gamma \sim 3)$ nonsalty developer succeeded in developing out the resist in the gaps, leaving no bridging or footing between lines, unlike the case for the slightly higher contrast $(\gamma \sim 3.5)$ TMAH developer. The observation that the nonsalty and salty developers had very different contrasts and yet produced such similar results suggested that contrast $\gamma$ is only a good indicator and not an absolute measure of the process performance.

Next, we measured the development rate of HSQ as a function of time to observe the self-limiting nature of the development process. HSQ (XR-1541 6\% solids, Dow Corning) was spin coated at $2 \mathrm{krpm}$ spin speed for $1 \mathrm{~min}$ onto $\mathrm{Si}$ substrates, resulting in a thickness of $126 \mathrm{~nm}$. To avoid thermally induced contrast reduction, we did not bake the resistcoated wafer. Furthermore, to avoid aging effects on the resist, ${ }^{13}$ the total processing time from spin coating to development was limited to 3 days. A Raith 150 EBL system was used to expose a dose matrix of $10 \times 80 \mu \mathrm{m}^{2}$ rectangles spanning a range of doses from 500 to $3000 \mu \mathrm{C} / \mathrm{cm}^{2}$ in a geometric progression with a multiplication factor of 1.05
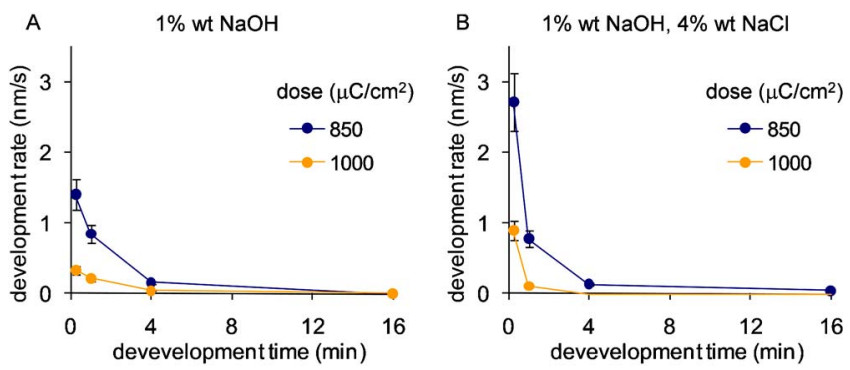

FIG. 4. (Color online) Plots of development rate vs development time for (a) the nonsalty developer and (b) the salty developer, showing for clarity only two representative exposure doses of 850 and $1000 \mu \mathrm{C} / \mathrm{cm}^{2}$. In both developers, the development rate rapidly decreased and stopped beyond 4 min of development. The initial development rate in (b) was noticeably higher than that in (a), indicating that the salty developer was more efficient at removing reaction products and enhancing dissolution.

(resulting in 38 rectangles) at $30 \mathrm{kV}$ acceleration voltage. The results at $100 \mathrm{kV}$ acceleration voltage have also shown similar trends. ${ }^{14}$ Several sets of dose arrays were exposed in the same run on the same wafer to reduce variations between separate experimental runs. We then cleaved the Si wafer to separate out different dose-array sets and developed each set for different amounts of time $(15 \mathrm{~s}, 1 \mathrm{~min}, 4 \mathrm{~min}$, and $16 \mathrm{~min}$ ) in the salty and nonsalty developer solutions. The thickness of the resist remaining in each rectangle of the dose arrays was measured using a surface profilometer to generate contrast curves. Finally, the development rate for each given exposure dose at a given development time was obtained by calculating the resist thickness removed in the exposed area divided by the development interval. For instance, the development rate at the $4 \mathrm{~min}$ development time $=\left(\mathrm{RTR}_{4 \min }-\mathrm{RTR}_{1 \min }\right) /(4 \min -1 \mathrm{~min})$, where $\mathrm{RTR}_{x \min }$ is the resist thickness remaining after $x$ min of development.

For clarity, Fig. 4 shows the development rate of only two different exposure doses of 850 and $1000 \mu \mathrm{C} / \mathrm{cm}^{2}$, although development rates for a dozen the other exposure doses were obtained and showed a similar trend. These doses were chosen as they were within the sloped region of the contrast curves for both salty and nonsalty developments. Note that the development rates for both developers were nonconstant but rapidly decreased to almost negligible rates beyond 4 min of development. Importantly, the initial development rates and the difference in the development rates between the 850 and $1000 \mu \mathrm{C} / \mathrm{cm}^{2}$ doses were larger for the salty compared to the nonsalty development. This result suggests that a high initial development rate might be an important indicator of good developer performance. Recent results ${ }^{14}$ have also shown a later-stage $(>10 \mathrm{~min})$ thinning effect that occurred only in the salty development. However, we did not observe such effects in the experiments reported in this article possibly due to differences in processing conditions, such as the total processing time, which was 3 days in our case compared to 1 day in Ref. 14. Nonetheless, from our experience, 3 days of total processing time was short enough to have no (or only a slight) effect on the results presented here. 

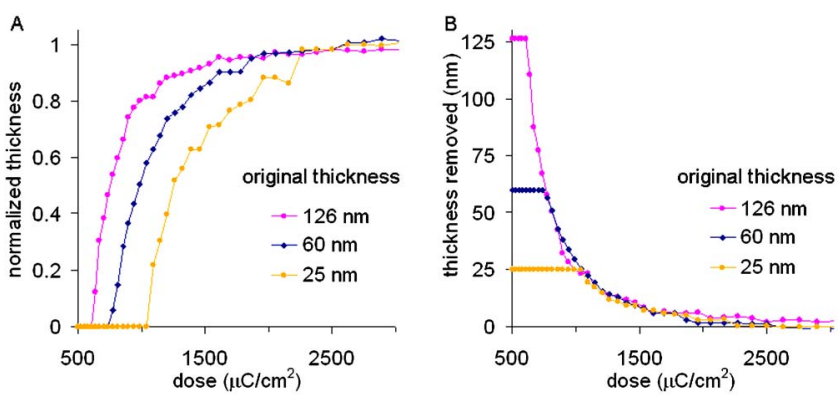

FIG. 5. (Color online) (a) Contrast curve plots of the normalized resist thickness remaining vs dose for three different resist thicknesses developed for $16 \mathrm{~min}$ in the nonsalty developer, suggesting that a thinner resist is less sensitive. However, plotting the same data as resist thickness removed vs dose in (b) shows that the data fall on a single curve (except, of course, for the low-dose saturation regions where the resist has been fully removed), indicating that regardless of the initial resist thickness, the thickness of the resist removed is constant for a given exposure dose.

The self-limiting nature of the development has been attributed to the occurrence of resist cross-linking during resist development that competes with the dissolution process. ${ }^{15,16}$ This cross-linking process adds to the cross-linking that occurred during resist exposure. A hypothesis explaining the possible reaction mechanism during development is as follows.

First, a hydroxide ion $\mathrm{OH}^{-}$reacts with the $\mathrm{Si}-\mathrm{H}$ functional group that may be attached to an unmodified HSQ molecule or a larger cross-linked molecule to form an ionized silanol group $\mathrm{Si}-\mathrm{O}^{-}$with the release of hydrogen gas, which is experimentally observed as the formation of bubbles during HSQ development. A plausible chemical equation for this reaction is as follows:

$$
\equiv \mathrm{Si}-\mathrm{H}+\mathrm{OH}^{-} \rightarrow \equiv \mathrm{Si}-\mathrm{O}^{-}+\mathrm{H}_{2} \text {. }
$$

The formation of a sufficient number of ionized sites per molecule would render the molecule soluble in the aqueous developer solution. On the other hand, larger molecules that are more heavily cross-linked during EBL exposure would remain insoluble due to the lower concentration of available $\mathrm{Si}-\mathrm{H}$ groups. Furthermore, ionized molecules that are not removed rapidly enough from the surface of the resist could also experience additional cross-linking to form $\mathrm{Si}-\mathrm{O}-\mathrm{Si}$ bonds via the following reaction:

$$
\begin{aligned}
\equiv & \mathrm{Si}-\mathrm{O}^{-}+\equiv \mathrm{Si}-\mathrm{O}^{-}+\mathrm{H}_{2} \mathrm{O} \rightarrow \equiv \mathrm{Si}-\mathrm{O}-\mathrm{Si} \equiv \\
& +2 \mathrm{OH}^{-} .
\end{aligned}
$$

The development eventually slows and comes to a stop due to the formation of extensive degree of cross-linking on the surface of the resist. ${ }^{15}$ This surface effect implies that for a given exposure dose above an onset dose, the thickness of the resist removed during development is fixed regardless of the original resist thickness. To show that this implication is indeed true, we measured contrast curves obtained from three different resist thicknesses $(126,60$, and $25 \mathrm{~nm})$. Figure 5(a) shows the resultant contrast curves plotted as the normalized resist thickness remaining versus exposure dose for the development in nonsalty developer for $16 \mathrm{~min}$. This

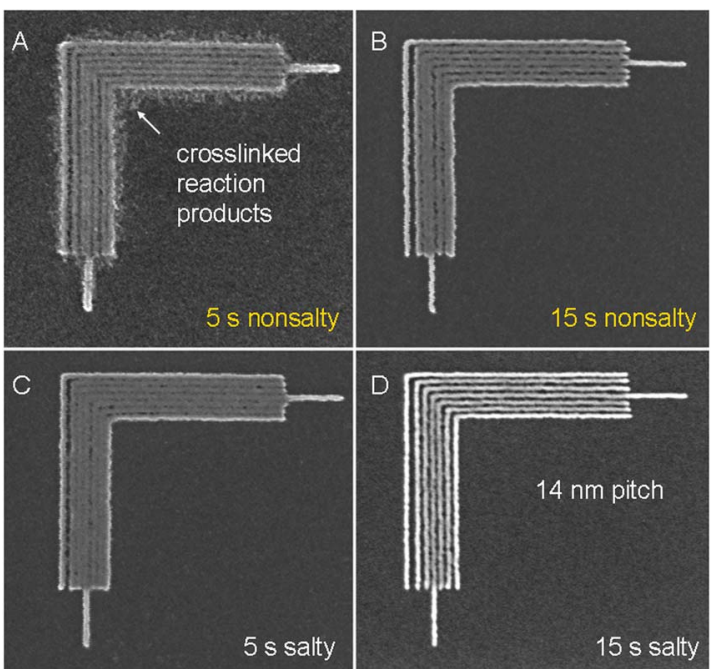

FIG. 6. (Color online) SEM images of 14-nm-pitch nested-L test structures patterned in 15-nm-thick HSQ using a line dose of only $5.5 \mathrm{nC} / \mathrm{cm}$ and developed for short amounts of time of 5 and $15 \mathrm{~s}$ in [(a) and (b)] nonsalty and $[(c)$ and (d)] salty developers. Samples were imaged in a Raith 150 EBL at $10 \mathrm{kV}$ acceleration voltage and $6 \mathrm{~mm}$ working distance. For the $5 \mathrm{~s}$ developments, the presence of scum in (a) and not in (c) confirms the higher initial development rates of the salty developer over that of the nonsalty developer. After $15 \mathrm{~s}$ of development, the 14-nm-pitch structures were already fully resolved in (d) but not in (b). The ability of the salty developer to quickly remove reaction products might be advantageous in reducing the occurrence of cross-linking during development.

plot seems to suggest that the resist sensitivity decreases with decreasing resist thickness. ${ }^{17}$ However, a more illuminating plot is shown in Fig. 5(b) where the same data were plotted as resist thickness removed versus exposure dose. Here, we see that all three different resist thicknesses lie on a single curve. Therefore, the contrast curve for a thinner resist can be obtained from contrast measurements of a thicker resist simply by extracting the relevant portion of the contrast curve.

Figure 6 shows SEMs of 14-nm-pitch nested-L test structures developed for only 5 and 15 s using the salty and nonsalty developers. We see by comparing Figs. 6(a) and 6(c) that the salty developer indeed had a higher initial development rate and more effective removal of reaction products compared to the nonsalty developer. The slower removal of reaction products in the nonsalty developer resulted in the appearance of scum around the nested-L structures. By comparing Figs. 6(b) and 6(d), we see that while an additional $10 \mathrm{~s}$ of development time resulted in clearly resolved 14-nm-pitch structures in the salty developer, the slower development of the nonsalty developer was unable to fully develop the resist in the gaps.

We have also observed that longer development times did not result in improvements in the pattern quality. Figures 7(a) and 7(b) show SEMs of 12-nm-pitch nested-L structures developed for $15 \mathrm{~s}$ and $4 \mathrm{~min}$, respectively, in the salty developer. It is indicated from the SEM images that a longer development did not improve the development of the resist between lines but served only to narrow the structures and 


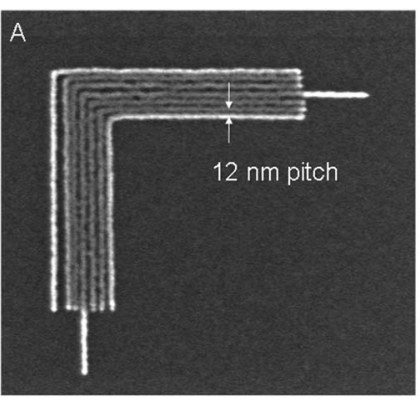

15 s develop

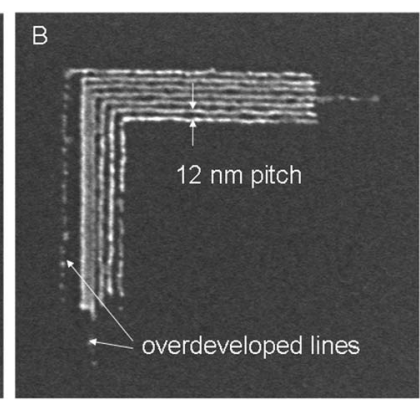

4 min develop
FIG. 7. SEM images of 12-nm-pitch nested-L structures patterned in 15-nm-thick HSQ using a line dose of $5.5 \mathrm{nC} / \mathrm{cm}$ and developed for (a) $15 \mathrm{~s}$ and (b) $4 \mathrm{~min}$ in the salty developer. Samples were imaged in a Raith 150 EBL system at $10 \mathrm{kV}$ acceleration voltage and $6 \mathrm{~mm}$ working distance. Even at this resolution, the $15 \mathrm{~s}$ development was sufficient to completely develop the resist in between the lines, while the 4 min development narrowed and overdeveloped some of the structures without improving removal of the resist in the gaps.

caused overdevelopment in the isolated lines, which had slightly lower doses due to proximity effects.

\section{DISCUSSION}

We first demonstrated that a 9-nm-pitch resolution could be achieved by exposure using a Raith 150-TWO EBL system even at a relatively low acceleration voltage of $10 \mathrm{kV}$. The Raith 150-TWO system in Dortmund, Germany, afforded a slight improvement in resolution $(9 \mathrm{~nm}$ pitch versus $10 \mathrm{~nm}$ pitch) over the Raith 150 system at the MIT campus.

We also showed in Figs. 2 and 3 that although the TMAH and nonsalty developers had similar contrast, their highresolution performance was different. This result suggested that the $\mathrm{Na}^{+}$ions were more effective in developing HSQ than the $\mathrm{TMA}^{+}$. Hence, although it is understood that the hydroxide ion $\left(\mathrm{OH}^{-}\right)$is the responsible reactant for HSQ development, the anions also play an important role during development. Guided by this result, one hypothesis for the development mechanism is as follows: during development of the slightly dosed regions in-between exposed structures, the surface of the resist becomes negatively charged due to the presence of ionized $\mathrm{Si}-\mathrm{O}^{-}$groups belonging to molecules on the resist surface. The positively charged anions are then attracted to the surface to screen the surface charges. The $\mathrm{TMA}^{+}$ions, being larger than the $\mathrm{Na}^{+}$ions, are less mobile and, thus, less effective at charge screening. Furthermore, the large $\mathrm{TMA}^{+}$ions and their low mobility might cause steric hindrance, thus reducing the access of the resist to $\mathrm{OH}^{-}$ions. As a result, the ionized $\mathrm{Si}-\mathrm{O}^{-}$groups have increased opportunity for cross-linking, which would further slow down the development and eventually forms footing/ bridging between structures. On the other hand, $\mathrm{Na}^{+}$ions are smaller, more mobile anions that are effective in screening the negatively charged resist surface, to hence allowing the negatively charged $\mathrm{OH}^{-}$ions to approach and completely develop the resist between the line exposed structures. The effect of adding $\mathrm{NaCl}$ to the $\mathrm{NaOH}$ developer was to therefore increase the concentration of $\mathrm{Na}^{+}$ions, and improving the charge screening to further increase the development rate in the lightly exposed regions. The effects of the $\mathrm{Cl}^{-}$ions are currently still unclear.

The observation of a higher initial development rate for the salty developer compared to the nonsalty developer in Fig. 4 supports the claim that improved charge screening caused by increased $\mathrm{Na}^{+}$concentration promotes access of $\mathrm{OH}^{-}$ions to the negatively charged surface and enhances development. This high initial development rate is beneficial for high-resolution EBL in thin films because it results in a rapid removal of reaction products, which would otherwise cross-link to form bridging/footing/scum between exposed structures.

\section{CONCLUSION}

We demonstrated the patterning of 4.5-nm-half-pitch structures using EBL exposure combined with a highcontrast salty development process. The development process of HSQ was investigated and shown to be a rapidly self-limiting process. The slowing and eventual stopping of development suggests that to achieve high resolution, the developer needs to quickly remove resist in the gaps between structures before the competing process of cross-linking occurs to form scum between exposed structures. Hence, a high initial development rate might be more important than a high contrast in determining the performance of a developer. Although the resolution of this EBL process of HSQ might be currently limited by the EBL tool, it is likely that the ultimate resolution would be determined by the resist performance. In future investigations on HSQ development, we will focus on testing the hypotheses of surface-charge formation and charge screening.

\section{ACKNOWLEDGMENTS}

The authors thank Henry I. Smith, James M. Daley, and Mark K. Mondol for helpful discussions. Patterning was done at MIT's shared scanning-electron-beam-lithography facility in the Research Laboratory of Electronics. One of the authors (J.K.W.Y.) would like to acknowledge his fellowship from A*STAR Singapore. This work was funded in part by Alfaisal University and King Abdulaziz City for Science and Technology. The work at Seoul National University was supported by the Frontier Research Program of Tera-Level Nanodevices (TND) funded by the Korean Ministry of Science and Technology (MOST).

${ }^{1}$ W. F. van Dorp, B. van Someren, C. W. Hagen, and P. Kruit, Nano Lett. 5, 1303 (2005).

${ }^{2}$ J. K. W. Yang and K. K. Berggren, J. Vac. Sci. Technol. A 25, 2025 (2007).

${ }^{3}$ W. Wu et al., Nano Lett. 8, 3865 (2008).

${ }^{4}$ P. Hashemi, M. Canonico, J. K. W. Yang, L. Gomez, K. K. Berggren, and J. L. Hoyt, ECS Trans. 16, 57 (2008).

${ }^{5}$ I. Bita, J. K. W. Yang, Y. S. Jung, C. A. Ross, E. L. Thomas, and K. K. Berggren, Science 321, 939 (2008).

${ }^{6}$ X. Yang, S. Xiao, W. Wu, Y. Xu, , K. Mountfield, R. Rottmayer, K. Lee, D. Kuo, and D. Weller, J. Vac. Sci. Technol. B 25, 2202 (2007).

${ }^{7}$ Y. F. Chen, H. F. Yang, and Z. Cui, Microelectron. Eng. 83, 1119 (2006).

${ }^{8}$ M. Haffner, A. Heeren, M. Fleischer, D. P. Kern, G. Schmidt, and L. W. Molenkamp, Microelectron. Eng. 84, 937 (2007). 
${ }^{9}$ V. Sidorkin, E. van der Drift, and H. Salemink, J. Vac. Sci. Technol. B 26, 2049 (2008)

${ }^{10}$ A. E. Grigorescu, M. C. van der Krogt, C. W. Hagen, and P. Kruit, Microelectron. Eng. 84, 822 (2007).

${ }^{11}$ B. Cord, J. K. W. Yang, H. Duan, D. C. Joy, J. Klingfus, and K. K. Berggren, "Limiting factors in sub-10-nm scanning-electron-beam lithography," J. Vac. Sci. Technol. B (these proceedings).

${ }^{12}$ As this manuscript was in preparation we received preliminary evidence of a similar result in HSQ using a $100 \mathrm{kV}$ Elionix EBL tool with similar processing conditions (courtesy of Yusuke Uchiyama and Ken Koseki).
${ }^{13}$ F. C. M. J. M. van Delft, J. Vac. Sci. Technol. B 20, 2932 (2002).

${ }^{14}$ S.-W. Nam, M. J. Rooks, J. K. W. Yang, K. K. Berggren, H.-M. Kim, M.-H. Lee, K.-B. Kim, J.-H. Shim, and D. Y. Yoon, "Contrast enhancement behavior of hydrogen silsesquioxane in a salty developer," J. Vac. Sci. Technol. B (these proceedings).

${ }^{15}$ H. S. Lee, J. S. Wi, S. W. Nam, H. M. Kim, and K. B. Kim, J. Vac. Sci. Technol. B 27, 188 (2009).

${ }^{16}$ G. M. Schmid, L. E. Carpenter, and J. A. Liddle, J. Vac. Sci. Technol. B 22, 3497 (2004).

${ }^{17}$ V. Sidorkin, A. Grigorescu, H. Salemink, and E. van der Drift, Microelectron. Eng. 86, 749 (2009). 